\title{
Cinq espèces nouvelles de Diptères Psychodidae Psychodinae du Liban
}

\author{
F. Vaillant 1 \\ Z. Moubayed ${ }^{2}$
}

Mots clés : espèces nouvelles, Diptera, Psychodidae, taxinomie, Liban.

Des représentants de 10 espèces de Psychodinae ont été recueillis au Liban ; 4 d'entre elles ont une vaste répartition en Europe et méme en dehors de ce continent ; 5 autres especes sont nouvelles et chacune d'entre elles est proche d'une espèce de la partie occidentale de l'Europe.

Five new species of Diptera Psychodidae Psychodinae from the Lebanon

Keywords : new species, Diptera, Psychodidae, Taxonomy, Lebanon.

Specimens of 10 species of Psychodinae were collected in the Lebanon : 4 of them have a wide range in Europe, which extends beyond this continent ; 5 other species are new and each one of these is close to a species from Western Europe.

A notre connaissance, il n'existe que deux documents concernant les Diptères de la sous-famille des Psychodidae Psychodinae représentés au Liban et ils sont très récents (Wagner 1980, 1984). L'un de nous a capturé dans ce pays des représentants de 10 espèces, dont 5 sont déjà connues.

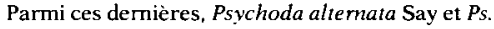
cinerea Banks sont cosmopolites et ont été transportées par l'homme sur tous les continents et dans presque toutes les îles des régions froides, tempérées et tempérées-chaudes du globe. Psychoda severini Tonnoir a un vaste territoire dans l'hémisphère boréal. Psychoda lativentris Berdén a une aire géographique très morcelée en Europe et en Asie. Bazarella jungi Wagner, probablement endémique de montagnes du Moyen-Orient, est proche de B. atra (Vaillant), qui est représentée dans presque toutes les hautes montagnes du pourtour de la Mediterranée, sauf pour la partie orientale de celle-ci. Des détails sont donnés dans une autre publication

1. Université Scientifique et Médicale de Grenoble. Laboratoire de Zoologie. 38402 Saint-Martin-d'Hères, Cédex.

2. Université Paul Sabatier. Laboratoire d'Hydrobiologie. 118, route de Narbonne, F. 31062 , Toulouse, Cédex.
(Moubayed 1986) concernant les localités dans lesquelles ces Diptères ont été capturés.

Les cinq autres espèces sont nouvelles. Voici la description des imagos máles pour quatre d'entre elles et celles des imagos des deux sexes pour la cinquième.

La nomenclature concernant les genitalia est celle de F. Vaillant 1982.

\section{Pericoma chlifasica n. sp.}

Imagos o (fig. 1-4): Espace interoculaire de 2,5 à 3 diamètres de facette. Suture interoculaire en $V$ très ouvert. Rangées obliques de facet tes de l'ceil formées successivement de 3.4.4.4.4... éléments. Rapports de longueur des articles antennaires: 30.32.27.25.24.24.24.25.25.23.23.22.20.17.16.25; une paire d'ascoïdes sur chacun des segments 6 à 13 ; le dernier article a un prolongement apical épais et très excentrique. Rapports de longueur des articles du palpe : 49.63.78.132. Rapport alaire : 2,50 ; angle médial : $163^{\circ}$; angle apical : $101^{\circ}$; la nervure $m_{2}$ forme un angle obtu à sa base. Tergite abdominal IX 
environ aussi long que large en vue dorsale ; bande sternale IX progressivement épaissie vers sa partie médiane ; plaque post-sternale très grande et à bords latéraux parallèles ; gonocoxite à apodème proximodorsal très caractéristique (fig. 3 ), grèle, avec un talon terminal. Gonostyle avec un a bec „épais. Cercopodes chacun avec 4 rétinacles ni denticulés ni ramifiés. Paramères remarquablement larges ; ductores étroits ; les uns et les autres rejoignent à leur extrémité une pièce transversale fortement chitinisée. Valvule subgénitale et valvule anale de forme classique.

\section{Longueur de l'aile : 2,9-3,1 mm.}

L'holotype et 7 paratypes ont été pris le 21.10.1981 à Chlifa, en bordure d'un ruisseau rapide formé par les eaux de captage de Yammoné, et entre 1100 et $1200 \mathrm{~m}$ d'altitude.

Pericoma chlifasica appartient, parmi les espèces du genre Pericoma, au groupe pseudoexquisita; elle est proche de $P$. corsicana Vaillant, endémique de la Corse, dont elle se distingue par la taille, particulièrement grande, de sa plaque post sternale et par la forme de ses paramères. Elle est encore plus proche de $P$. platistyla Wagner, de Turquie, dont elle se distingue par une taille plus grande de sa plaque poststernale et par la largeur plus grande de ses paramères. D'autre part ni $P$. corsicana, ni $P$. platystyla n'ont les apodèmes proximo-dorsaux de leurs gonostyles rétrécis.

\section{Pericoma litanica n. sp.}

Imago o (fig. 5 et 6): Espace interoculaire de 4 diamètres de facette. Suture interoculaire en $U$ très ouvert. Rangées obliques de facettes de l'oil formées successivement de 1. 3 ou 4.4.4.4.4.... éléments. Rapports de longueur des articles antennaires: 30.33 .29 .25 .23 .23 .23 .23 .23 .22 .20 .18 .18 .15 .14 .22 ; une paire d'ascoïdes sur chacun des segments 6 à 13 ; dernier article semblable à celui de l'espèce précédente, $P$. chlifasica. Rapports de longueur des trois premiers articles du palpe: 42.54.68. Rapport alaire : 2,72 ; angle médial : $189^{\circ}$; angle apical : $100^{\circ}$. Tergite abdominal IX aussi long que large ; bande sternale IX grèle; plaque poststernale grande, à échancrure anguleuse et à bords latéraux légèrement convergents vers l'arrière; gonocoxites incurvés et plus de 2 fois plus longs que larges en vue dorsale; gonostyles à courbure presque régulière en vue dorsale ; cercopodes avec chacun 4 à 6 rétinacles ni denticulés, ni ramifiés ; paramères très larges, mais arrondis sur leur bord latéropostérieur : ductores rectilignes et fortement élargis à leur exırémité postérieure

Longueur de l'aile variable et comprise entre 2,2 et $2,6 \mathrm{~mm}$.

L'holotype et I paratype ont été pris le 17.5.1982 à Hermel ; le lieu de capture est à $650 \mathrm{~m}$ d'altitude et il est en bordure d'un cours d'eau affluent de l'Oronte et qui provient de la principale source de celui-ci. Trois autres mâles ont été trouvés entre 900 et $1000 \mathrm{~m}$ en bordure du Ghozayel, affluent du Litani, le 29.3.1982.

Pericoma litanica appartient au groupe diversa des Pericoma; elle est particulièrement proche de $P$. granadica Vaillant, de la péninsule ibérique et du Maroc ; toutefois, chez cette dernière espèce, le tergite abdominal IX est plus large que long, la plaque poststernale est courte, les gonocoxites sont presque rectilignes, les paramères sont étroits et fortement incurvés. $P$. litanica est également proche de $P$. kugleri Wagner, d'Israël ; elle s'en distingue par les caractères suivants : ses gonocoxites sont plus longs et plus grèles que ceux de cette espèce ; la plaque poststernale a ses bords latéraux convergents vers l'arrière et non parallèles; enfin ses ductores sont plus larges que ceux de $P$. kugleri.

\section{Panimerus hermelinus n. sp.}

Imago $\sigma$ (fig. 7 et 8) : Espace interoculaire de 4 diamètres de facette. Suture interoculaire légèrement incurvée. Rangées obliques de facet tes de l'œil formées successivement de 2.3.4.4.4.... éléments. Rapports de longueur des 3 premiers articles antennaires : 66.28 .19 ; scape 3 fois plus long que large ; pédicelle 1,6 - 1,7 fois plus long que large, avec une forte sailiie distale ventrale garnie de chètes. Corniculi claviformes et progressivement rétrécis depuis leur $6 / 7$ distal jusqu'à leur base ; ils sont presque aussi longs que le scape antennaire. Aile anguleuse a son apex; rapport alaire: 2,36; angle médial : $186^{\circ}$ : angle apical : $61^{\circ}$. Tergite abdominal IX plus large que long en vue dorsale; bande sternale IX très épaisse et ciliée dans sa partie médiane ; plaque poststernale avec deux lobes très écartés l'un de l'autre et deux sclérites médians distaux ; gonostyles longs, grèles, incurvés seulement 

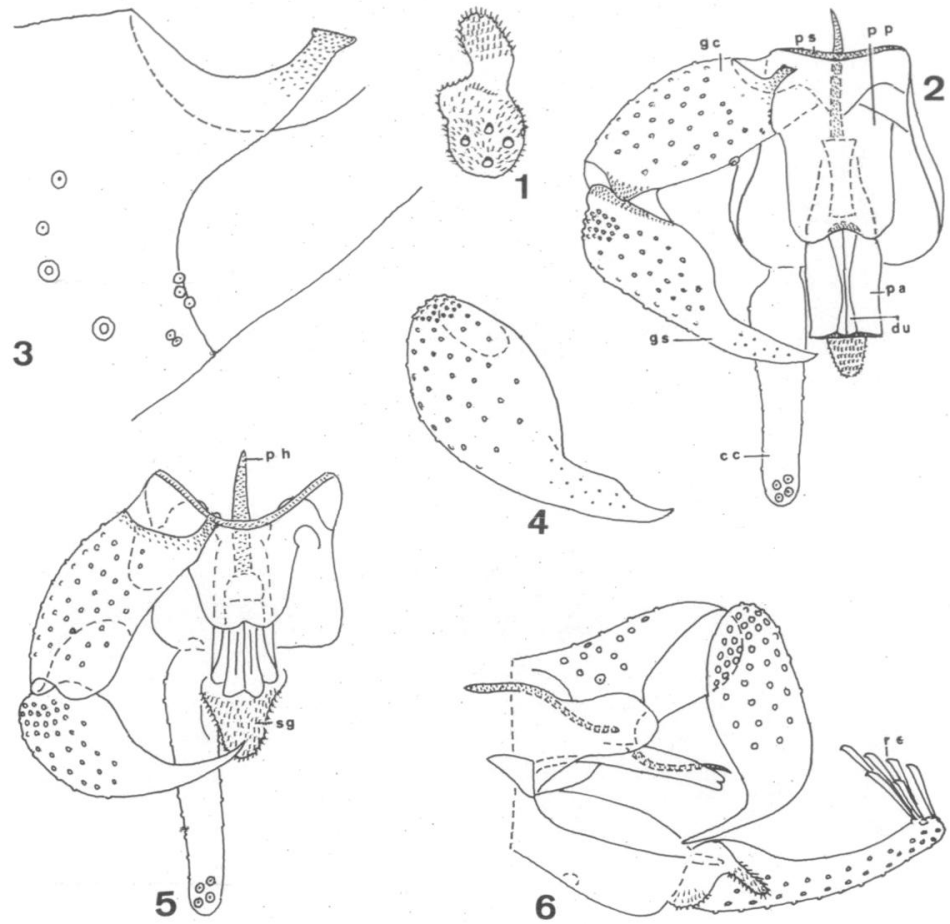

Fig. 1 à 4 : Pericoma chlifasica n. sp., imago $\sigma .1$ : dernier article antennaire : 2 : genitalia, face dorsale ; $3:$ partie proximale du gonocoxite gauche, détail, face dorsale ; 4 : gonostyle gauche, vue oblique.

Fig. 5 et 6 : Pericoma litanica n. sp., imago $\sigma .5$ : genitalia, face dorsale ; 6 : genitalia, profil ; le gonopode et le cercopode gauches ont été enlevés.

cc : cercopode, du : ductor, gc : gonocoxite, gs : gonostyle, pa : paramère, ph : phallapodème, pp : plaque poststemale, ps : bande sternale IX, re : rétinacle, sg: valvule subgénitale.

sur leur 1/4 distal et pointus à leur apex; chaque cercopode a 6 ou 7 rétinacles ni denticulés ni ramifiès; paramères plus longs que le tergite abdominal IX et régulièrement incurvés ; ductores anguleux et non denticulés; phallapodème circulaire et en cuiller.

Longueur de l'aile : $2,5 \mathrm{~mm}$.
Un seul spécimen, l'holotype, dont les antennes sont incomplètes et dont les palpes manquent, a été capturé le 17.5.1982 à Hermel à $650 \mathrm{~m}$ d'altitude en même temps que le type de Pericoma litanica.

Panimerus hermelinus fait manifestement partie du groupe notabilis parmi les nombreuses espèces de Panimerus. Elle est particulièrement proche de 

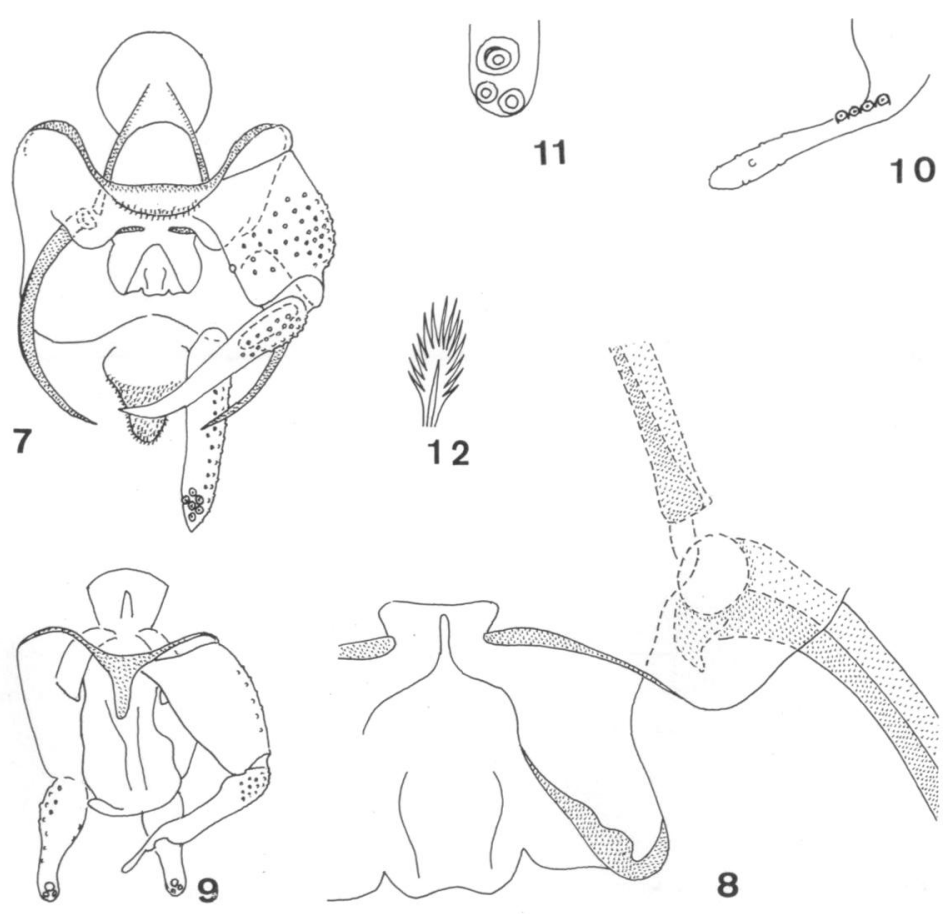

Fig. 7 et 8 : Panimerus hermelinus n. sp., imago $\sigma^{\circ} .7:$ genitalia, face dorsale $: 8$ : paramère droit à sa base, ductor droit et partie droite de la plaque poststernale, détail, face dorsale.

Fig. 9 à 12 : Tonnoiriella filistylis n. sp., imago $\sigma .9$ : genitalia, face dorsale ; 10 : partie distale du gonostyle droit, face postérieure ; 11 : partie distale du cercopode gauche, face dorsale ; les rétinacles sont supposés enlevés ; 12 : partie distale d'un des petits rétinacles.

$P$. denticulatus Krek, des Balkans, mais en diffère par son angle apical $\left(61^{\circ}\right.$ au lieu de $\left.79^{\circ}\right)$, par le nombre des rétinacles de chaque cercopode 6 ou 7 au lieu de 12) et surtout par la forme élancée de ses gonostyles. $P$. hermelinus est encore plus proche de $P$. freidbergi Wagner, d'Israël, mais, chez cette dernière espèce, la bande sternale IX n'est ni épaissie en son milieu, ni ciliée, les gonocoxites sont épaissis à leur base, les paramères sont plus longs que pour l'espèce du Liban et arrondis à leur extrémité : enfin le phallapodème est beaucoup plus petit chez $P$. freidbergi que chez $P$. hermelinus.

\section{Tonnoiriella filistylis $n$. sp.}

Imago ơ (fig. 9 à 13): Espace interoculaire de 6 diamètres de facette. Suture interoculaire en $V$ très ouvert. Rangées obliques de facettes de l'ceil 
formées successivement de 2.3.3.3.4.... éléments. Rapports de longueur des 5 premiers articles antennaires : 24.23.19.20.22 ; scape et pédicelle plus lar. ges que longs. Rapports de longueur des 3 premiers articles du palpe: 34.40 .38 . Aile avec des parties enfumées, comme chez toutes les espèces de Tonnoiriella, et à bord apical acuminé; rapport alaire : 2,95 ; la nervure $\mathrm{m}_{2}$ est libre à sa base ; si on joint par une droite la fourche radiale et l'extrémité de

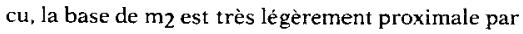
rapport à cette droite ; angle apical : $105^{\circ}$. Tergite abdominal IX à bords latéraux convergents vers l'arrière: bande sternale IX avec un épaississement médian très accusé et formant une longue saillie : plaque poststernale scindée en deux pièces en forme de croissant; gonocoxites chacun avec un prolongement dorso-interne acuminé : gortostyles très particuliers, allongés, brusquement rétrécis aux $2 / 3$ de leur longueur, puis avec une partie grèle, garnie sur sa face externe d'une série de 4 soies très rapprochées et situées chacune sur une forte saillie ; chaque cercopode a un grand et deux petits rétinacles fortement ramifiés à leur extrémité ; paramères et ductores soudés pour former une plaque asymétrique, avec un a bec »distal tourné vers la gauche ; phallapodème en cuiller, à bords latéro-antérieurs anguleux.

Longueur de l'aile : $2,1 \mathrm{~mm}$.

Un seul spécimen, l'holotype, dont les antennes et les palpes sont incomplets, a été pris le 17.5.1982 à Hermel à $650 \mathrm{~m}$ d'altitude avec le type de Pericoma litanica.

Tonnoiriella filistylis se distingue de toutes les autres espèces du même genre par la forme très particulière de sa bande sternale IX et par celle, encore plus remarquable, de ses gonostyles.

\section{Psychoda lebanica n. sp.}

Imago $\sigma$ (fig. 14-16): Espace interoculaire d'un seul diamètre de facette. Pas de suture interoculaire. Rangées obliques de facettes de l' $\propto$ eil formées successivement de 2.4.4.4.4... éléments. Rapports de longueur des 8 premiers segments antennaires: 23.24.20.24.26.27.25.24; ascoüdes à 3 branches, dont l'une est récurrente. Labelles du labium chacun avec 3 denticules. Rapport alaire : 2,83 ; angle médial : $97^{\circ} ; r_{5}$ aboutit à l'apex acuminé de l'aile. Tergite abdominal IX bien plus large que long. Bande sternale IX peu incurvée, très longue et rétrécie seulement sur les côtés. Plaque poststernale asymétrique et arrondie sur son bord postérieur ; cercopodes à double courbure. L'aedeagus, asymétrique, se pro longe, dans sa partie postérieure, par une seule pièce digitiforme, qui provient vraisemblablement de la fusion des deux ductores. Phallapodème légèrement déprimé latéralement.

Longueur de l'aile : $1,7 \cdot 1,8 \mathrm{~mm}$.

Imago $९$ (fig. 17-19) : Aile comme celle du mâle. Plaque subgénitale bilobée, comme celle de Psychoda lobata Tonnoir, mais la nacelle génitale se prolonge en arrière par une longue tige fortement chitinisée et très caractéristique.

De nombreux spécimens des deux sexes, dont l'holotype, ont été pris à Anjar, Chamsine, entre 900 et $1000 \mathrm{~m}$ d'altitude, en bordure du Ghozayel, qui est un affluent du Litani ; malheureusement les antennes et les palpes de tous les exemplaires étaient absents ou incomplets.

Les imagos $\sigma^{*}$ de Psychoda lebanica se rapprochent particulièrement de celles de Ps. lobata Tonnoir ; de même les femelles des deux espèces ont des caractères voisins. Toutefois, chez le or de Ps. lobata, il y a une suture interoculaire, l'angle médial de l'aile est inférieur à $90^{\circ}$, il y a un seul paramère, qui est très court et fortement chitinisé, et la partie digitiforme de l'aedeagus est épaisse et courte. Chez la $Q$ de $P_{s}$. lobata, la plaque subgénitale est arrondie sur les côtés, au lieu d'avoir les bords droits, et la nacelle génitale ne se prolonge pas par une tige fortement chitinisée.

Ps. lebanica se rapprocherait, semble-t-il, encore plus de $P_{S}$. alternicula Quate, qui est signalée d'Israël par R. Wagner et redécrite par lui. Toutefois, chez l'imago $\sigma$ de Ps. alternicula, les gonopodes sont, dans $l^{2}$ ensemble, plus trapus que pour Ps. lebanica, et, chez l'imago $\varnothing$, les lobes de la plaque subgénitale sont pointus à leur extrémité, alors qu'ils sont arrondis pour Ps. lebanica.

\section{Travaux cités}

Moubayed (Z.) 1986. - Recherches sur la faunistique, l'écologie et la zoogéographie de trois réseaux hydrographiques du Liban : l'Assi, le Litani et le Beyrouth. Thèse d'Etat, $496 \mathrm{p}$. Toulouse.

Tonnoir (A.) 1922. - Synopsis des especes europèennes du genre Psychoda (Diptères) Ann. Soc. Ent. Belg., 62: 49.88. 


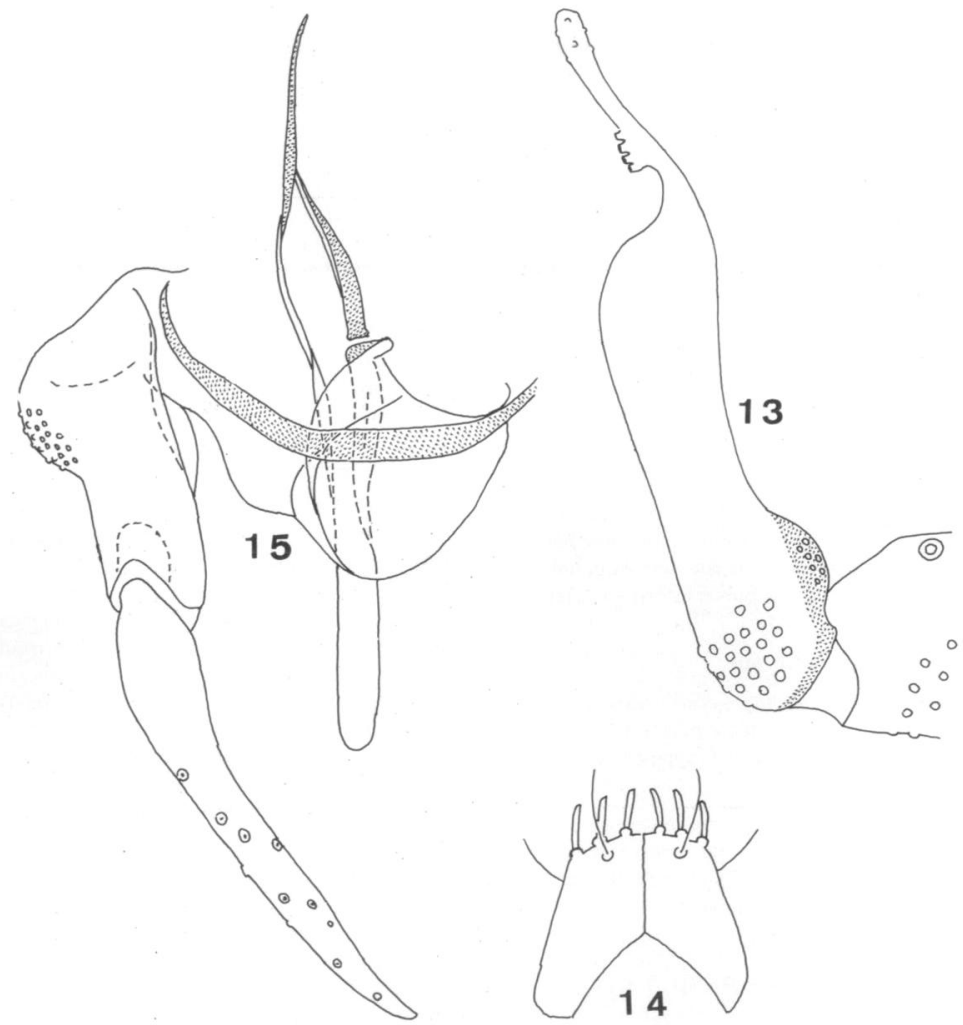

Fig. 13 : Tonnoiriella filistylis $\mathrm{n} . \mathbf{s p}$., imago o ; gonostyle droit, détail, face dorsale.

Fig. 14 et $15:$ Psychoda lebanica $n$. sp., imago $\sigma .14$ : labelles du labium, face ventrale : 15 : genitalia, face dorsale : tergite abdominal IX et cercopodes n'ont pas été figurés. 


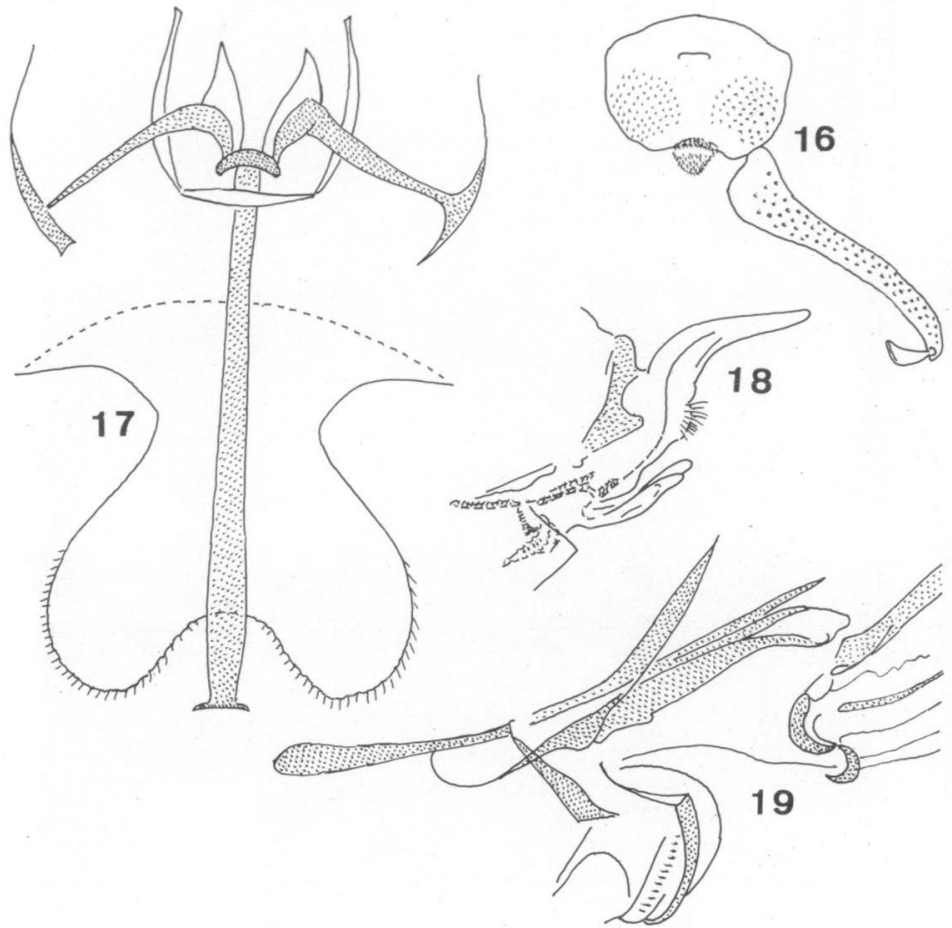

Fig. 16 : Psychoda lebanica n. sp., imago o ; tergite abdominal IX et cercopode gauche, face ventrale.

Fig. 17 à $19:$ Psychoda lebanica n. sp., imago $९ .17$ : plaque subgénitale et nacelle génitale, face dorsale ; $18:$ genitalia, profil ; 19 : nacelle génitale, détail, profil.

Vaillant (F.) 1971-1983. - Psychodidae Psychodinae in Lindner, E. : Die Fliegen der palaearktischen Region, 9d, Fischer, Stuttgart, 358 p., $120 \mathrm{Pl}$

Vaillant (F.) 1982. - Homologies entre les pièces génitales mâles de quelques Diptères Nématocères. Ann. Soc. Ent. Fr. (N.S.), 18 (3) : $419-425$.

Wagner (R.) 1980. - Pericoma becharreense n. sp., eine neue libanesische Psychodide aus der Sammlung des naturhistorischen Museums Wien. Ann. Naturhist. Mus. Wien, $83: 639 \cdot 640$.

Wagner (R.) 1984. - Bazarella jungi n. sp., eine neue Psychodide aus dem Libanon (Diptera : Psychodidae). Ann. Naturhist. Mus. Wien, $86: 81+83$.
Wagner (R.) \& Salamanna (G.). 1984. - Psychodinae of Sardinia III : Further records and descriptions of new species (Diptera Psychodidae). Boll. Soc. ent. ital., Genova, 116 (1-3) : 47-55

Wagner (R.). - Uber einige Psychodiden aus der Turkei (Diptera, Psychodidae), sous presse.

Wagner (R.). - On a small collection of Psychodidae (Diptera) from Israel, sous presse. 